\title{
Multifunctional conjugated polymer nanoparticles for photoacoustic-based multimodal imaging and cancer photothermal therapy
}

\author{
Xiaoju Men and Zhen Yuan* \\ Cancer Center, Faculty of Health Sciences \\ University of Macau, Macau SAR 999708, P. R. China \\ *zhenyuan@umac.mo
}

Received 23 December 2018

Accepted 15 January 2019

Published 20 February 2019

\begin{abstract}
Photoacoustic imaging (PAI) is a hybrid imaging method based on photoacoustic (PA) effects, which is able to capture the structure, function, and molecular information of biological tissues with high resolution. To date, therapeutic techniques under the guidance of PAI have provided new strategies for accurate diagnosis and precise treatment of tumors. In particular, conjugated polymer nanoparticles have been extensively inspected for PA-based cancer theranostics largely due to their superior optical properties such as tunable spectrum and large absorption coefficient and their good biocompatibility, and abundant functional groups. This mini-review mainly focuses on the recent advances toward the development of novel conjugated polymer nanoparticles for PA-based multimodal imaging and cancer photothermal therapy.
\end{abstract}

Keywords: Conjugated polymer nanoparticles; photoacoustic imaging; photothermal therapy; multimodal imaging.

\section{Introduction}

Biomedical imaging has exhibited its unbeatable advantages for mapping the structural and functional information of tumor tissues and for imagingguided cancer therapy. ${ }^{1,2}$ Meanwhile, nanotechnologies have led to the development of novel contrast agents for various molecular imaging methods such as fluorescence imaging, ${ }^{3}$ magnetic resonance imaging (MRI), ${ }^{4}$ computed tomography $(\mathrm{CT}){ }^{5}$ positron emission tomography (PET) ${ }^{6}$ and single photon emission computed tomography (SPECT). ${ }^{7}$ Interestingly, PAI is a unique high-resolution imaging technique, which can reveal the anatomy and function information of tumor with high optical contrast. ${ }^{89}$ However, the sensitivity of label-free PAI is dominated by the endogenous contrast, which may not be specific enough for cancer theranostics. By contrast, the utilization of nanoprobes

${ }^{*}$ Corresponding author.

This is an Open Access article published by World Scientific Publishing Company. It is distributed under the terms of the Creative Commons Attribution 4.0 (CC-BY) License. Further distribution of this work is permitted, provided the original work is properly cited. 
can improve the PAI contrast for high-accuracy cancer detection and imaging-guided cancer treatment. ${ }^{10}$ More importantly, the PAI probes can also serve as photothermal agents for photothermal therapy (PTT), which is recognized as a powerful cancer theranostics tool due to its high selectivity, low systemic toxicity, and negligible drug resistance. ${ }^{11,12}$

To date, nanomaterials have made significantly scientific breakthroughs in the fields of optics, electromagnetism, chemistry, and biology and medicine. ${ }^{13-16}$ Specifically, different nanoparticles have been produced and examined for improving the imaging contrast and enhancing the efficacy of cancer PPT, which consist of small-molecule dyes,${ }^{17}$ carbon dots ${ }^{18}$ metal nanoparticles, ${ }^{19}$ semiconductor quantum dots, ${ }^{20}$ and up-conversion nanoparticles. ${ }^{21}$ Among all nanoprobes available, conjugated polymer nanoparticles $(\mathrm{CPNs})$ are one of the most attractive candidates when applied to detection and treatment of cancer because of their excellent optical properties, including the tunable spectrum, large absorption coefficient, good photostability, good biocompatibility, and abundant functional groups. ${ }^{22-24} \mathrm{CPNs}$ have been utilized as multifunctional agents for vascular imaging, tumor imaging, cell tracking, and drug release tracking. ${ }^{25-27}$

Most recently, therapeutic techniques under the guidance of PAI have provided new strategies for accurate diagnosis and precise treatment of tumors. The development of multifunctional nanoprobes that integrate imaging diagnosis and imaging-guided treatment will reduce the number of treatments for patients and improve the tumor diagnostic accuracy and efficiency of treatment. ${ }^{28,29}$ In particular, CPNs have exhibited the extraordinary ability as a flexible nanoplatform for in vivo $\mathrm{PAI}$ of tumors, in which the strong $\mathrm{PA}$ signal enhanced by CPNs is largely due to their large optical absorption coefficient and high nonradiative quantum efficiency determined by the molecular structures of conjugated polymers. ${ }^{30,31}$ In addition, CPNs have also been inspected as theranostic agents for PA-based multimodal imaging and for PTT of cancer with the high photothermal conversion efficiency. ${ }^{32}$ This short review mainly focuses on the recent development of CPNs for PA-based multimodal imaging and PAI-guided cancer PTT.

\section{Preparation of Conjugated Polymer Nanoparticles}

The preparation methods for multifunctional CPNs mainly consist of the self-assembly method, miniemulsion, and nano-reprecipitation (Fig. 1). ${ }^{33}$ The self-assembly method denotes the spontaneous formation of nanostructures of conjugated polymers and functionalized materials under agitation, whereas the mini-emulsion and nano-reprecipitation methods imply dissolving a synthesized high molecular polymer in an organic solvent followed by interacting with water. During the mini-emulsion process, the nanoparticles are produced from emulsified droplets of the polymer and consequently an organic solvent that is immiscible with water is generally required. By contrast, in the nanoreprecipitation process, the nanoparticles are formed by precipitation of the polymer when they are rapidly mixed with water, and therefore an organic solvent that is miscible with water is required. However, the organic solvent is finally removed under high temperature. Interestingly, the preparation approaches can generate significant influence on the sizes of CPNs. For example, the mini-emulsion method is usually utilized to prepare large-size nanoparticles with the diameter ranged from 40 to $500 \mathrm{~nm}$, whereas the nano-reprecipitation method is for the production of small-size nanoparticle $(5-50 \mathrm{~nm})$.

In addition, the molecular weight also has a large impact on yield CPNs size. The high molecular weight of conjugated polymers always causes the poor solubility, preventing subsequent nanoprecipitation. Further, functional groups can provide additional influence on the size of CPNs. Importantly, the size of the nanoparticle is also strongly dependent on the starting concentration of the conjugated polymer, in which the higher the conjugated polymers starting concentration is, the larger the diameter should be. Previous studies showed that the size of CPNs has significant influence on the absorption spectrum and absorption coefficient. ${ }^{34}$ They discovered that small-size nanoparticles can result in the severe folding of the polymer skeleton, the destroyed conjugate length, and the absorption spectrum with a blue shift. They also observed that the spectrum of organic nanoparticles can be adjusted within a certain range by adjusting the size and molecular weight of organic nanoparticles without changing the structure of organic molecules. As a result, a suitable design 
Nano-precipitation Method

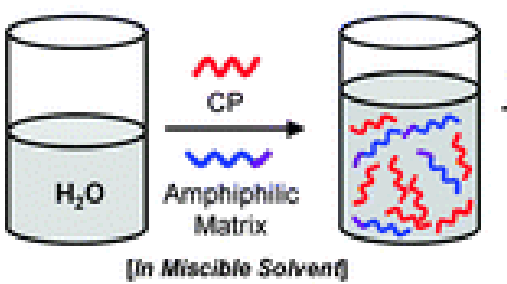

Ultrasonication

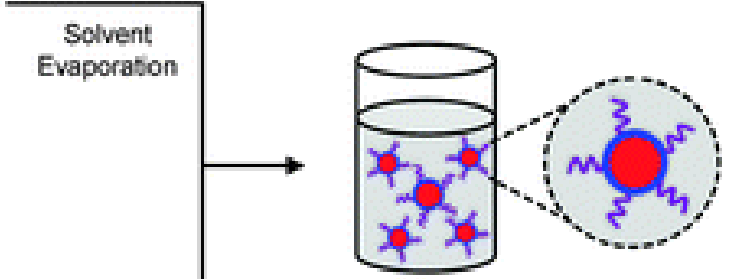

Mini-emuision Method

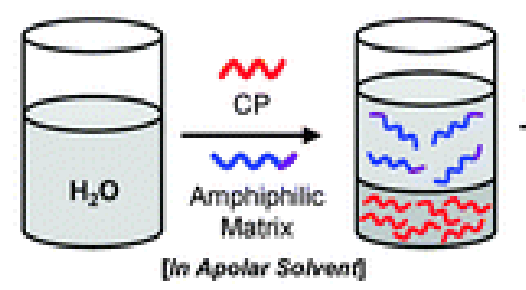

Ultrasonication

Solvent

Evapcration

[on Apolar Solvend

\section{Self-assembly Method}
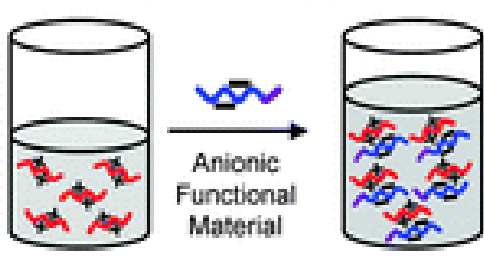

Stirring

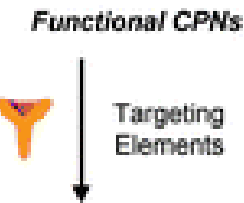

Cationic $\mathrm{CP}$ aqueous

Source: Reference 33, Copyright 2013, The Royal Society of Chemistry.

Fig. 1. Schematic representation of methods for preparing conjugated polymer nanoparticles.

should be optimized prior to the synthesis of multifunctional CPNs. Lots of multifunctional groups are applied to achieve the collaborative functions such as poly (styrene-g-ethylene oxide) (PS-PEG$\mathrm{COOH}$ ), poly(styreneco-maleicanhydride) (PSMA), and 1,2-distearoyl-snglycero-3-phosphoethanolamine-N-(polyethylene glycol) (DSPEPEG).

\section{Conjugated Polymer Nanoparticles for PA-Based Multimodal Imaging}

\subsection{Conjugated polymer nanoparticles for PAI}

In the past decade, CPNs have been well studied as contrast agents for enhanced PAI. More importantly, a rational design of molecular or nanoparticle structures is able to amplify PA signal intensity, leading to high-sensitivity tumor detection. Various strategies have been developed for the increase of PA intensity using CPNs such as introducing low-bandgap diketopyrrolopyrrole (DPP)-based conjugated polymers. ${ }^{29}$ In particular, PIID-TBT CPNs have been developed and used for enhanced PAI of blood vessels, ${ }^{35}$ in which PIID-TBT CPNs as the contrast agent can exhibit a more comprehensive characterization of biological tissues. CPNs can also serve as contrast agents for real-time PA vascular imaging of sentinel lymph node (Fig. 2). ${ }^{36}$

Interestingly, multilayered $\mathrm{CPNs}_{-} \mathrm{SiO}_{2}$ were also synthesized, which can simultaneously amplify fluorescence and PA signals for in vivo tumor imaging (Fig. 3(a)). ${ }^{37}$ They discovered that CPNs$\mathrm{SiO}_{2}$ exhibited higher PA intensity, which was about 1.4 higher than that from uncoated CPNs. In addition, semiconducting polymers with self-quenched fluorescence were also produced as contrast agents for enhanced PAI. ${ }^{38}$ Duo to the self-quenched process, 1.7-fold PA amplification of conjugated polymers for the corresponding CPNs. 


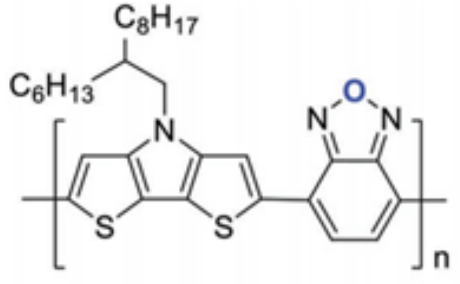

PDTPBD

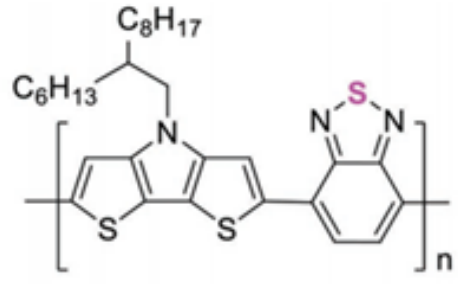

PDTPBT

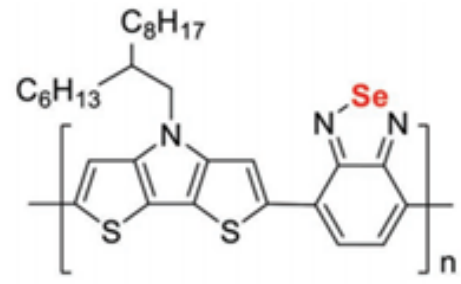

PDTPBSe

(a)
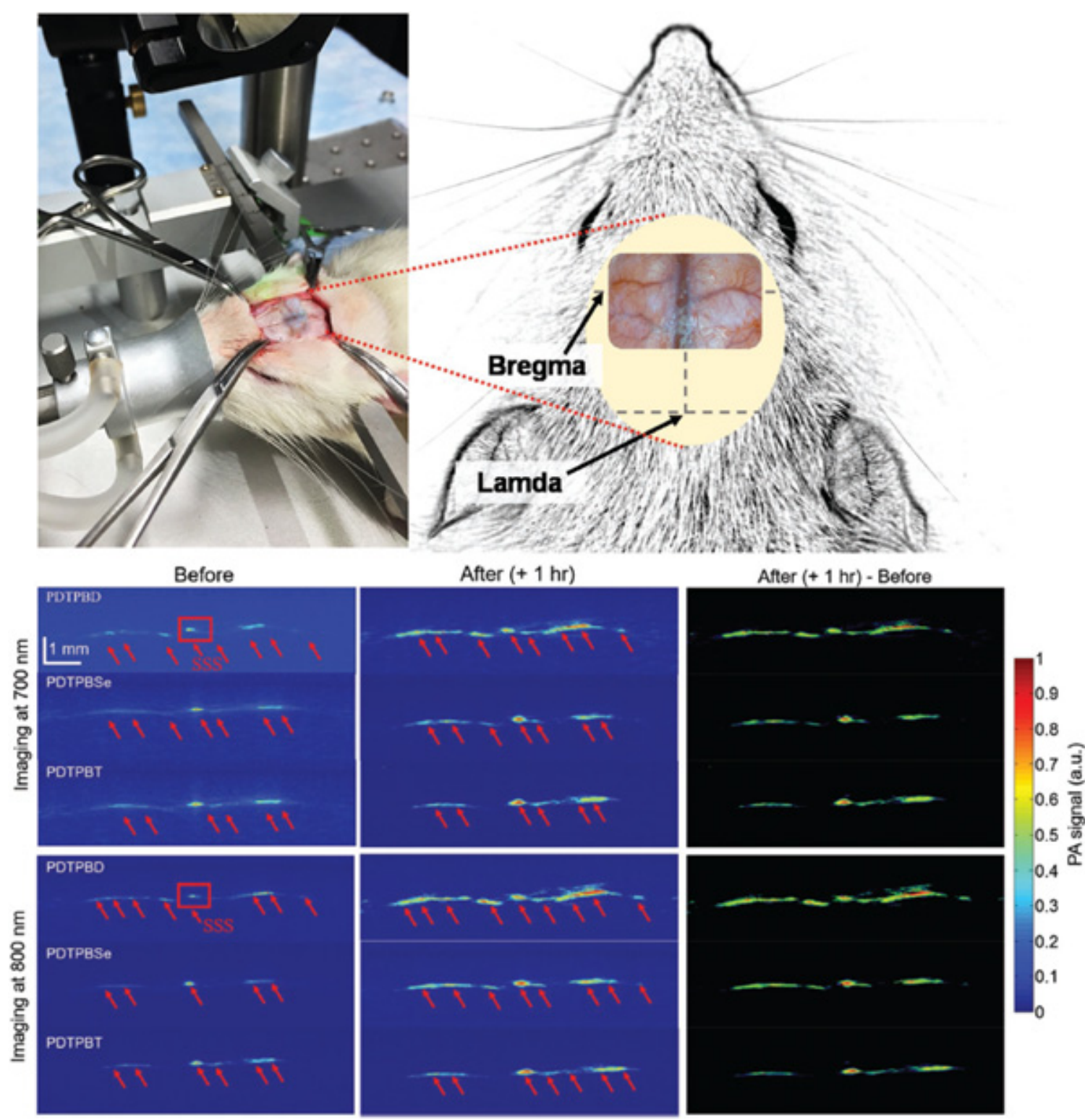

(b)

Source: Reference 36, Copyright 2018, Wiley-VCH.

Fig. 2. PAI of rat brain vasculature structures using CPNs at 700 and $800 \mathrm{~nm}$, respectively. 

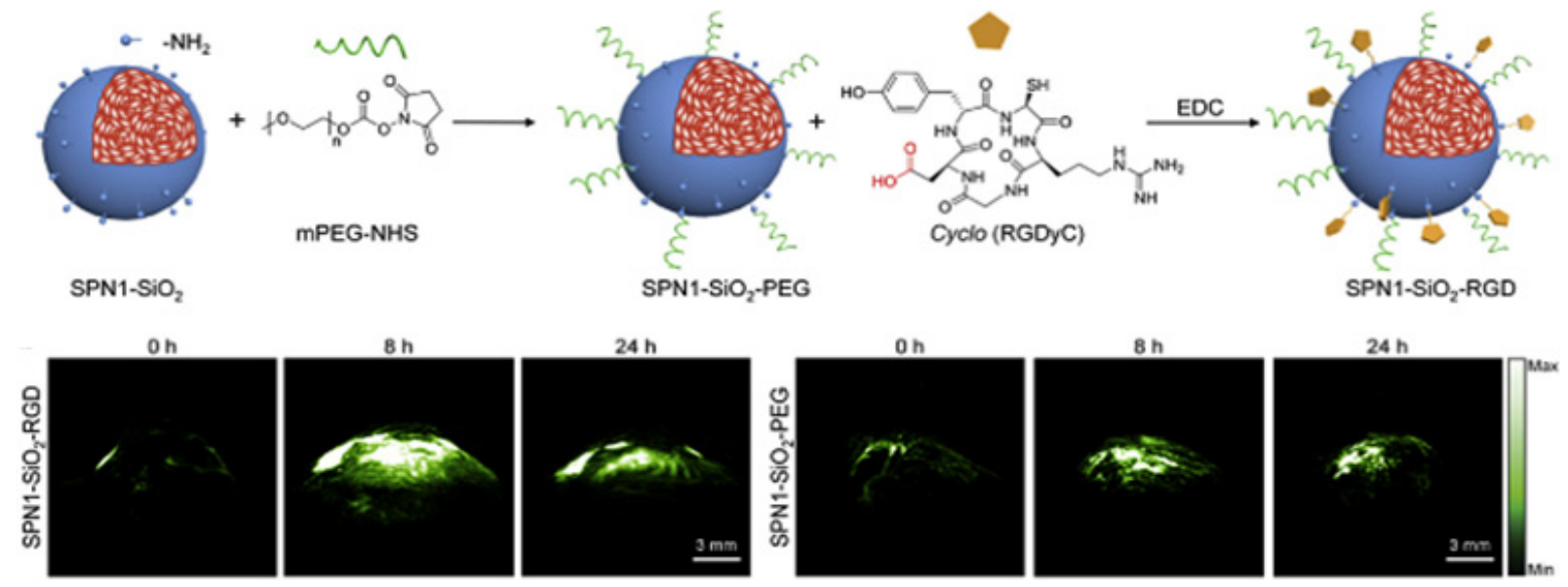

(a)
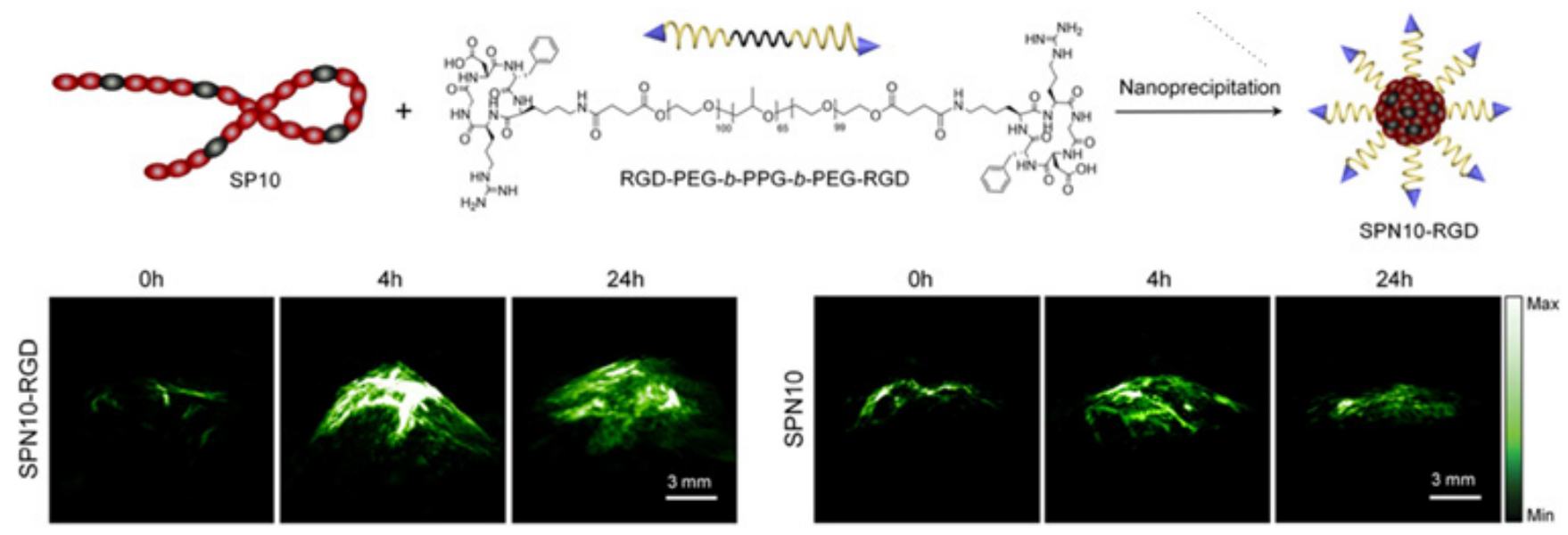

(b)

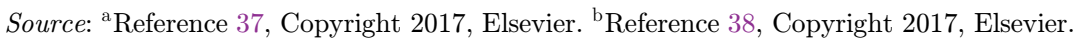

Fig. 3. (a) PA images of tumor at 0,8 and $24 \mathrm{~h}$ post-injection of $\mathrm{CPNs}-\mathrm{SiO}_{2} \cdot{ }^{\mathrm{a}}$ (b) $\mathrm{PA}$ images of tumor after injection of CPN10-RGD or SPN10. ${ }^{\text {b }}$

Through the combination of cyclic-RGD, the PA intensity can increase 4.7 -fold in living mice.

\subsection{Conjugated polymer nanoparticles for dual-modal PAI and fluorescence imaging}

Besides PA molecular imaging, fluorescence imaging (FLI) also exhibits its unbeatable advantages in mapping cellular level events such as gene and protein expressions and cancer cells. To take advantages of the complementary information from PAI and FLI, an optimized scheme has been developed, which use CPNs as dual-modal contrast agents for enhanced PAI and FLI. For example, Gao et al. fabricated multifunctional Au clustersfunctionalized BSA-modified CPNs for PA and fluorescence tumor imaging in vivo (Fig. 4a). ${ }^{39}$ Both the fluorescence and PA signals were able to be detected after the injection of Au cluster-functionalized BSA-modified CPNs in the living animal body. This method further demonstrated that BSAmodified CPNs can be an excellent dual-modal nanoprobe for PAI and FLI. In addition, the amphiphilic optically active CPNs SPA2-PEG2 was also constructed for in vivo PAI and FLI (Fig. 4b) ${ }^{40}$ The PA signals were 3 times higher than the background at the tumor site after the injection of SPA2-PEG2. Compared with CPNs prepared by 


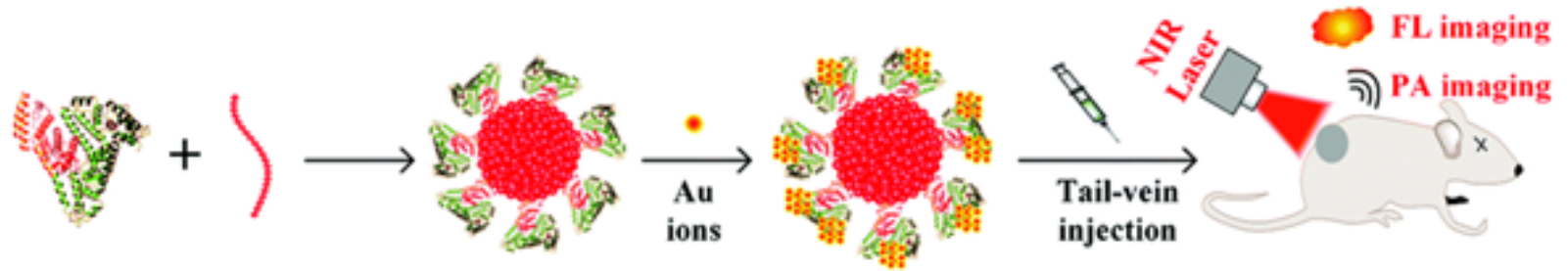

BSA

CP

BSA-modified CP nanoplatform
Au clusters-functionalized BSA-modified CPNs
In vivo tumor PA/FL dual-modal imaging
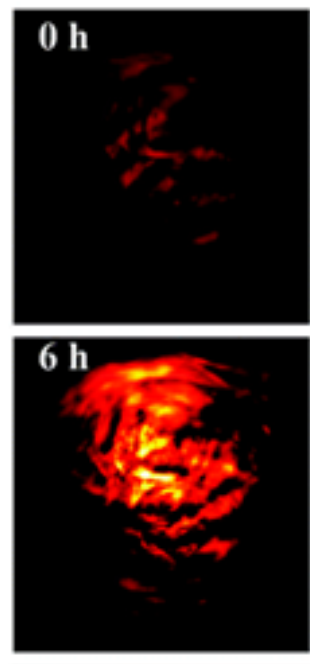

Low

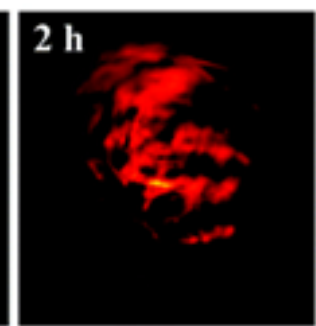

\section{$12 \mathrm{~h}$}
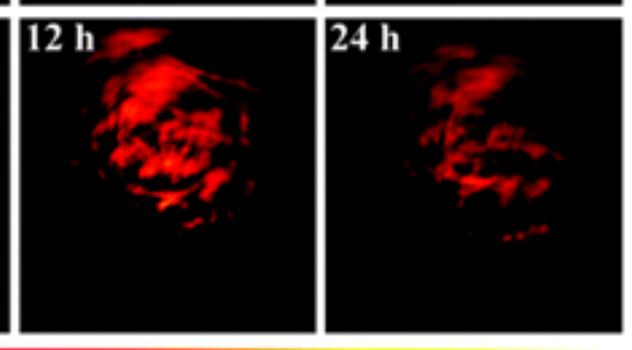

High
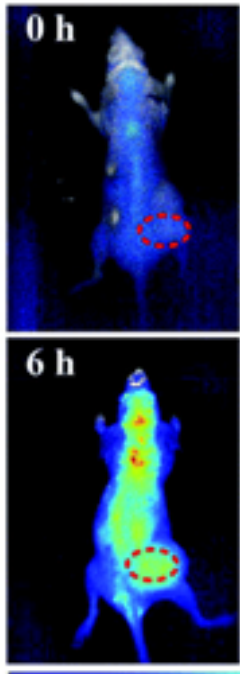

Low
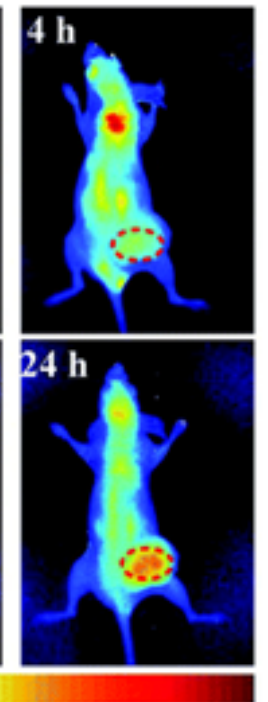

High

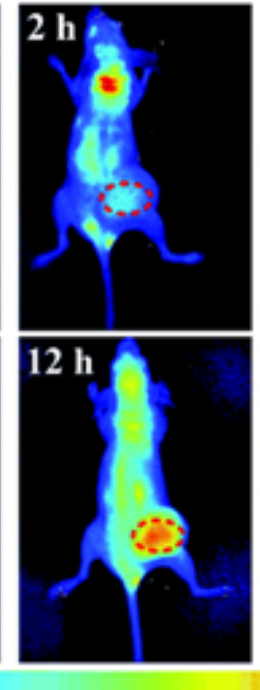

(a)

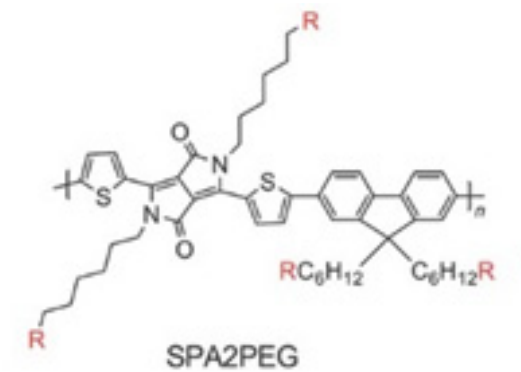

SPA2PEG
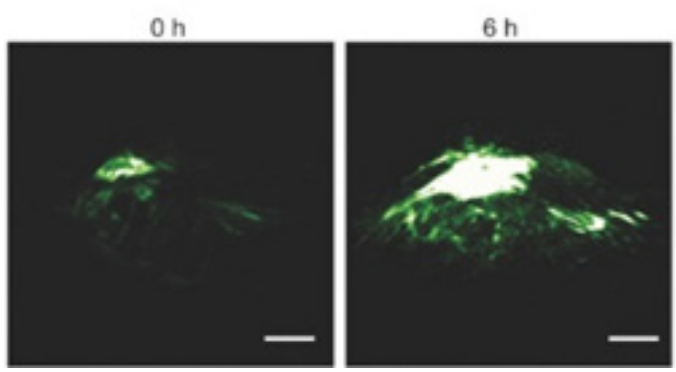

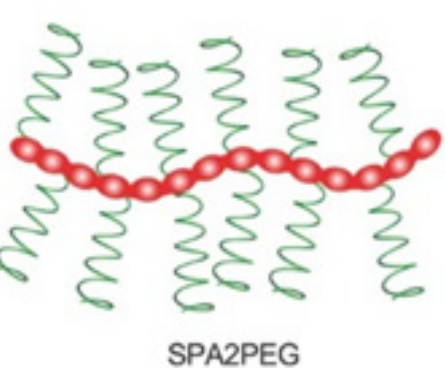

SPA2PEG

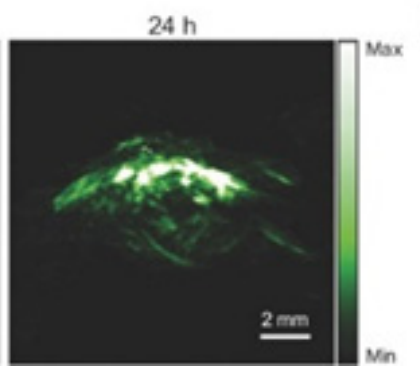

(b)
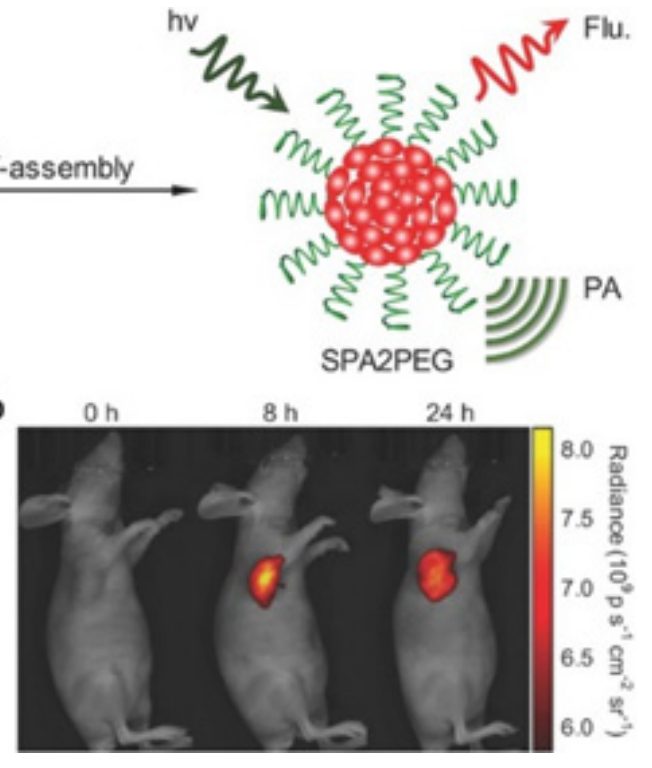

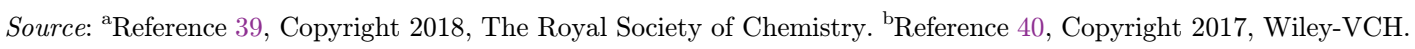

Fig. 4. (a) Au clusters-functionalized BSA-modified CPNs for FLI and PAI of tumor in vivo. ${ }^{\text {a }}$ (b) Real-time PAI and FLI of tumors after systemic administration of SPA2-PEG2 in tumor bearing mice. ${ }^{\mathrm{b}}$ 
nanoprecipitation, SPAs can generate higher fluorescence quantum yields with similar PA brightness, indicating that SPAs are more utilitarian for optical imaging applications. Further, the PEG chain of SPA2-PEG2 can result in reducing reticuloendothelial uptake and promoting EPR effect.

\section{Conjugated Polymer Nanoparticles for PAI-Guided PTT or Photo- thermal-Based Cancer Treatment}

The development of multifunctional CPNs will improve the detection accuracy and treatment efficiency of cancer. Meanwhile, the integration of non-invasive PAI and PAI-guided tumor therapies is essential to improve the survival rate of cancer patients. PTT strongly depends on the use of photothermal (PT) agents such as CPNs, whose photothermal conversion efficiency can be up to $50 \%$. In 2017 , a conjugated polymer with high-efficiency near-infrared (NIR) absorption was synthesized by using a band-theory to control the donor and acceptor of the conjugated polymer. ${ }^{34}$ High-sensitivity PAI-guided PTT was performed for in vivo cancer theranostics based on the designed CPNs (Fig. 5(a)). In addition, to improve the photothermal conversion efficiency of CPNs, Wang et al. designed a novel exogenous nanoprobe based on donor-acceptor

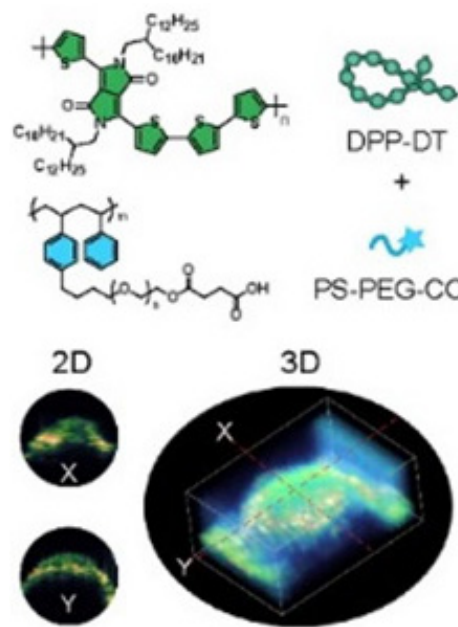

Photoacoustic Imaging
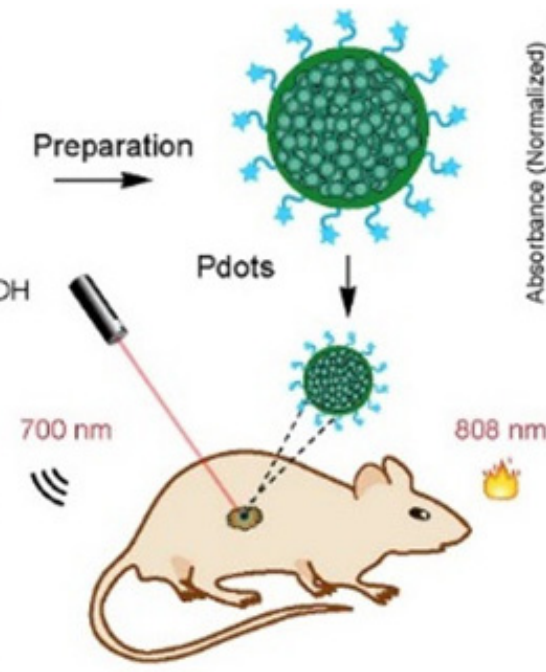

Photothermal Therapy
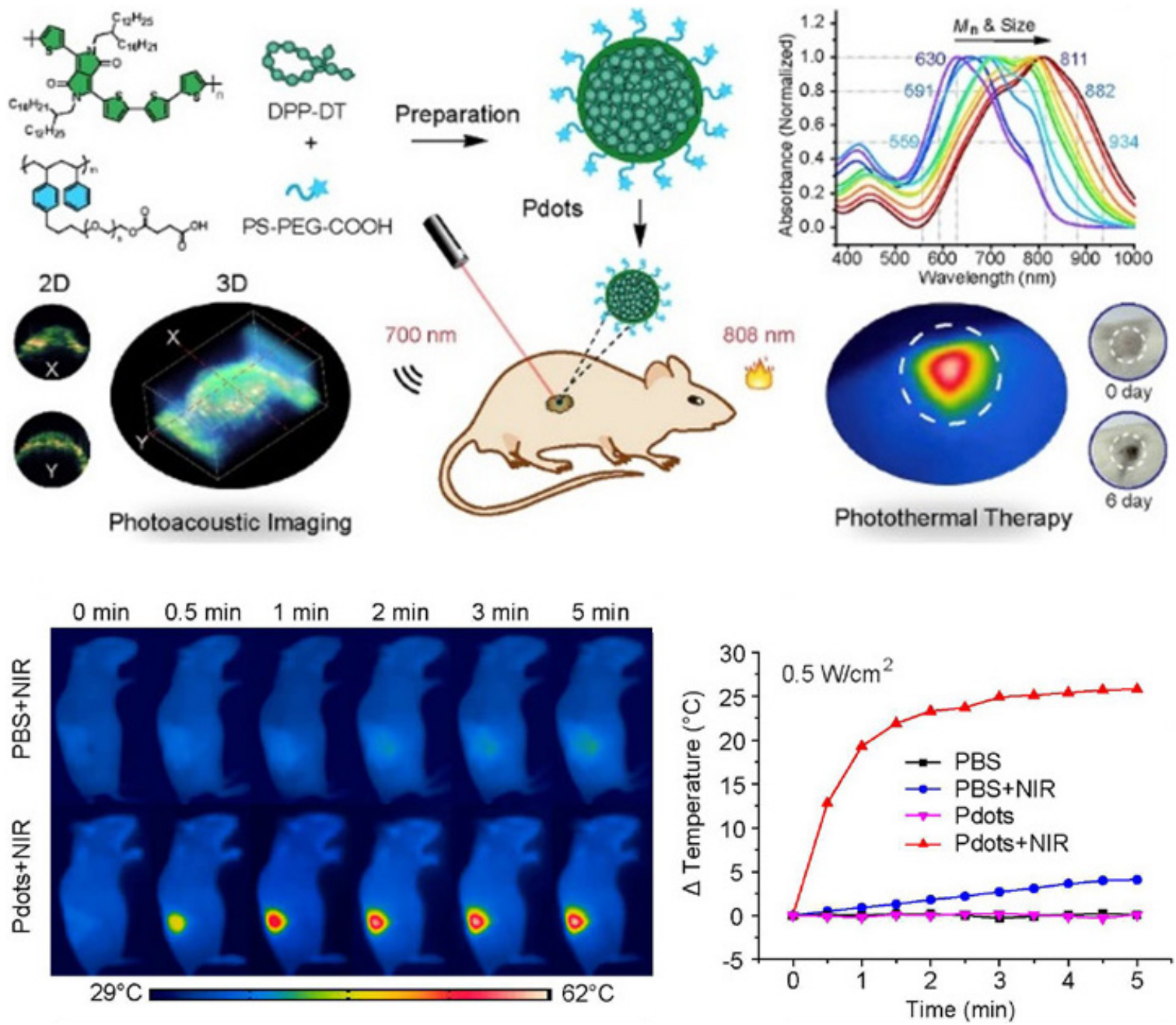

(a)

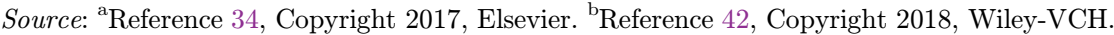

Fig. 5. (a) DPP-DT CPNs as contrast agents for PAI guided PTT. ${ }^{\mathrm{a}}$ (b) PAI and PTT of brain tumor. ${ }^{\mathrm{b}}$ 

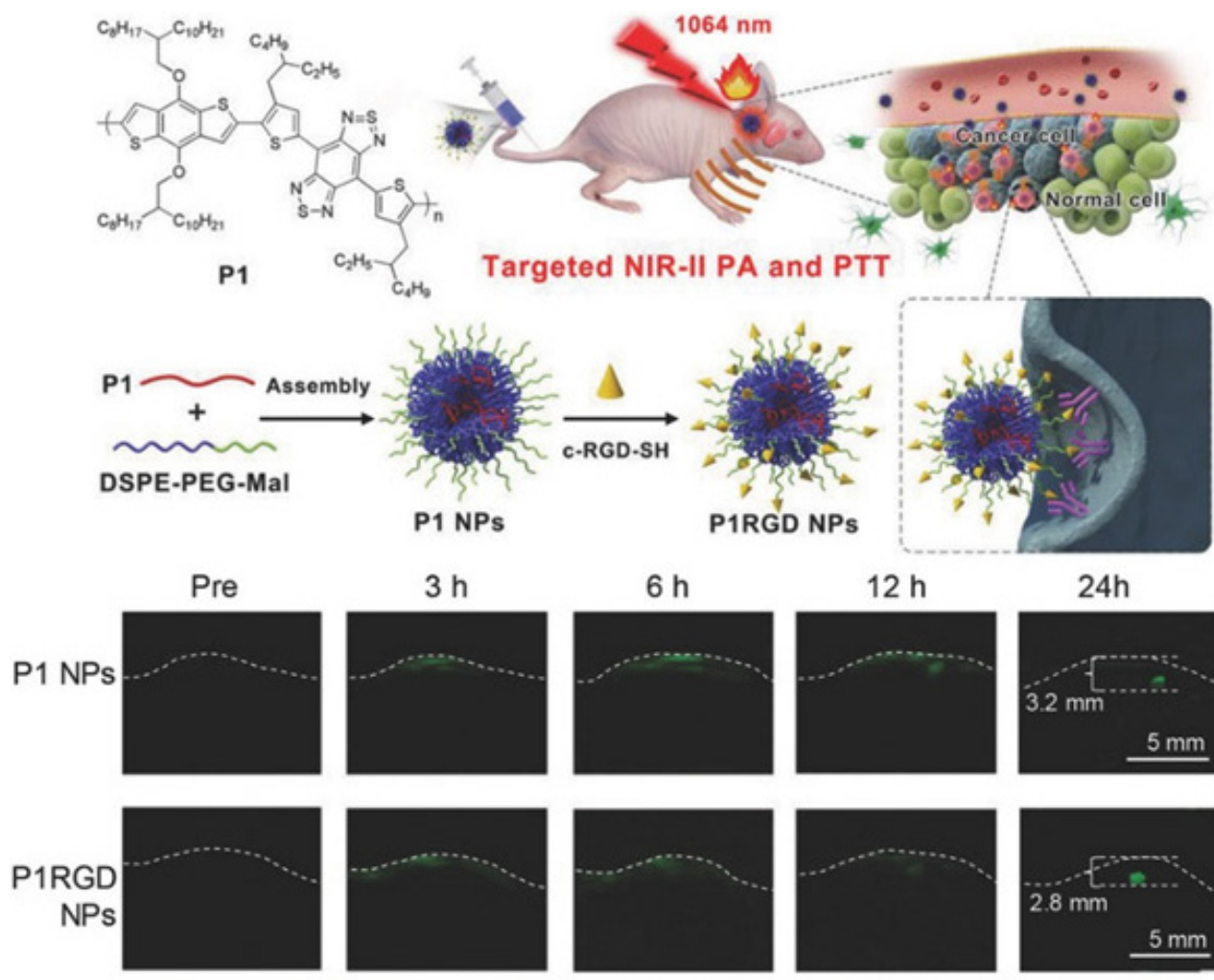

$6 \mathrm{~h}$

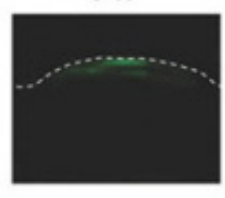

$12 \mathrm{~h}$

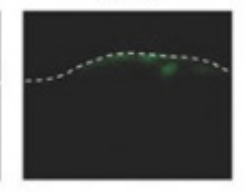

$24 \mathrm{~h}$
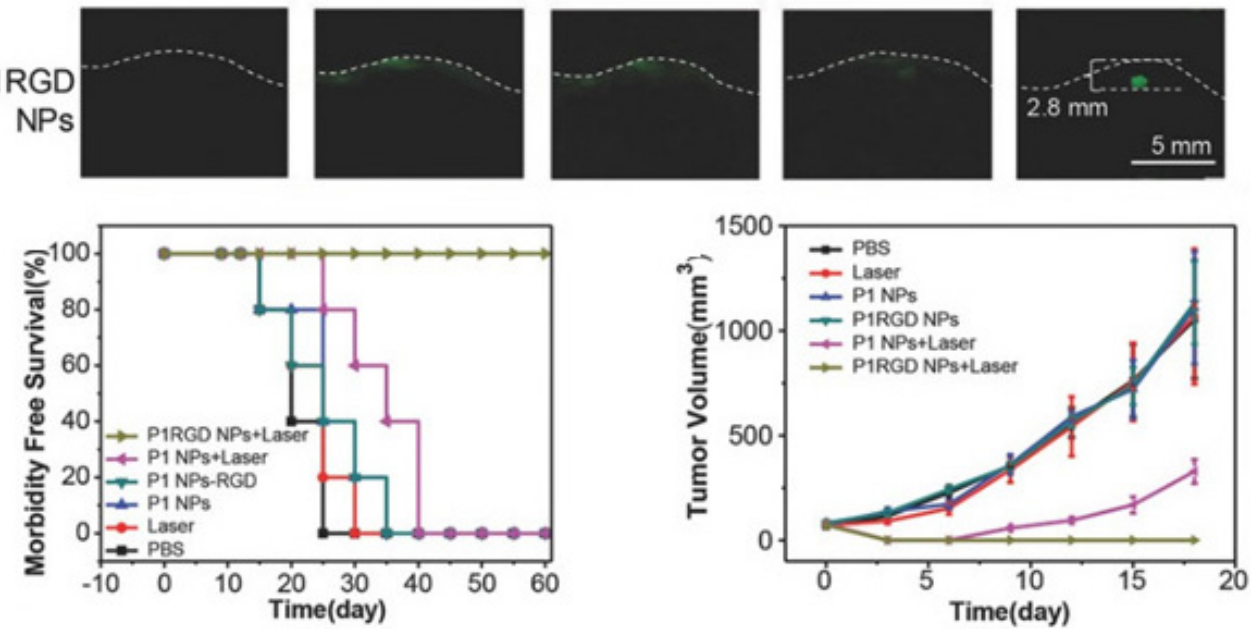

(b)

Fig. 5. (Continued)

CPNs, ${ }^{41}$ which have a high optical absorption coefficient and a low fluorescence quantum efficiency in the NIR window. Further, Liu's group first utilized CPNs to develop a brain tumor treatment paradigm for PAI guided PTT in the second near-infrared (NIR-II) window. ${ }^{42}$ Comparing to the NIR light at $808 \mathrm{~nm}$, the $1064 \mathrm{~nm}$ laser can effectively penetrate through skull and scalp, leading to a low value of therapeutically necessary power density. Due to the decoration of cyclo (Arg-Gly-Asp-D-Phe-Lys(mpa)) (c-RGD) on the surface of CPNs, CPNs can selectively target the tumor cells, leading to an ultra-high signal contrast to the background and significantly improvement therapeutic efficacy in brain tumor model (Fig. 5(b)).

\subsection{Conjugated polymer nanoparticles for PTT and chemotherapy}

Chemotherapy is the primary therapeutic method for cancer ablation, which suffers from low therapeutic efficacy, drug resistance of cancer tissues and side effects. Polymer-loaded drugs has the advantages of better biocompatibility, controlled drug release, higher drug payloads, and improved drug solubility. Therefore, compared with PTT or chemotherapy alone, drugs-loaded CPNs for concurrent 


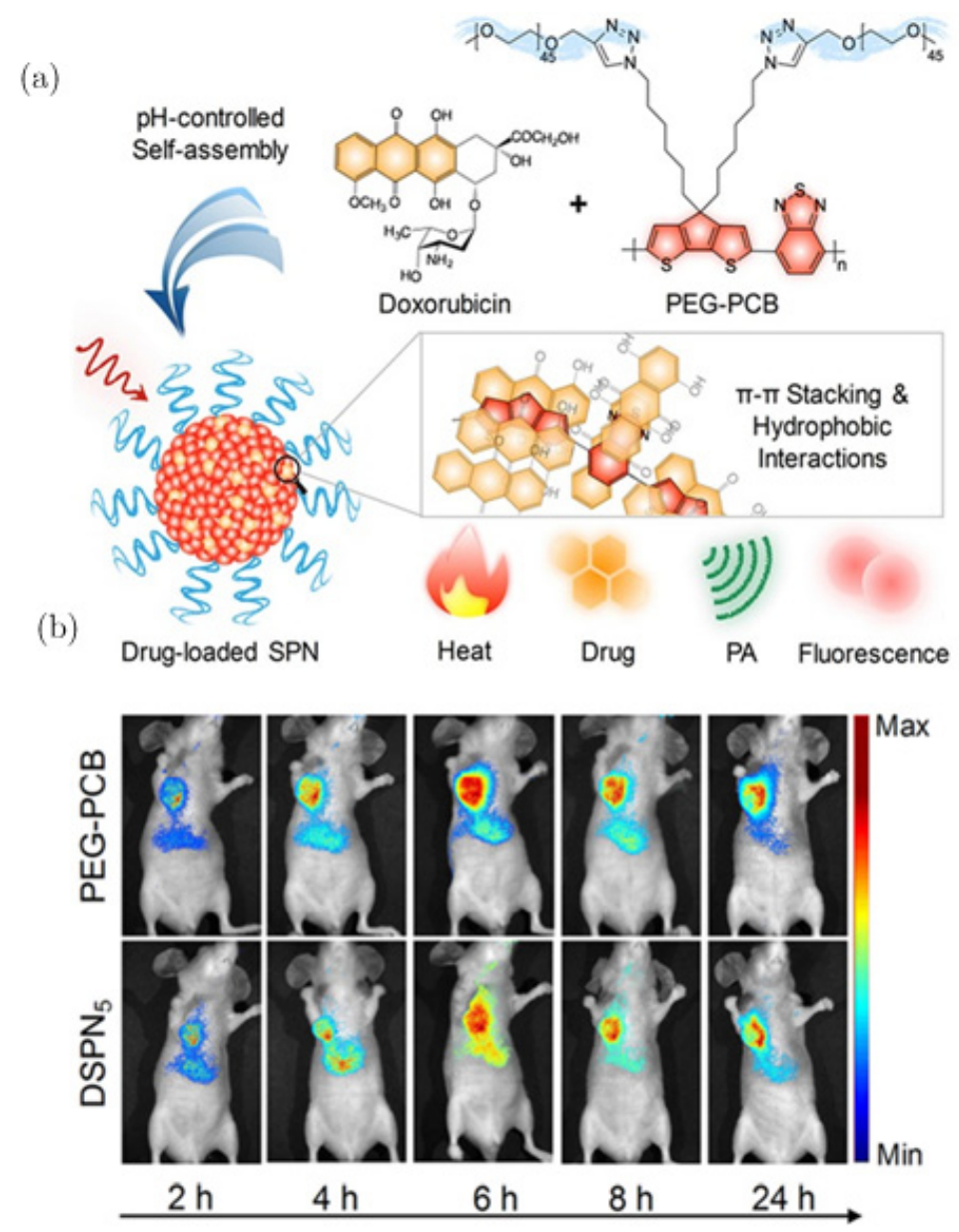

(c)
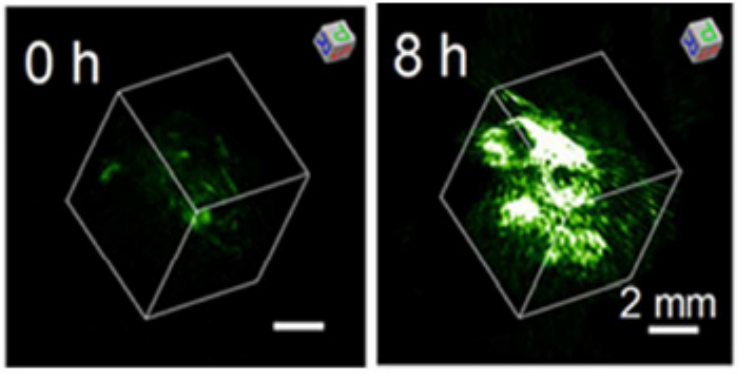

(d)
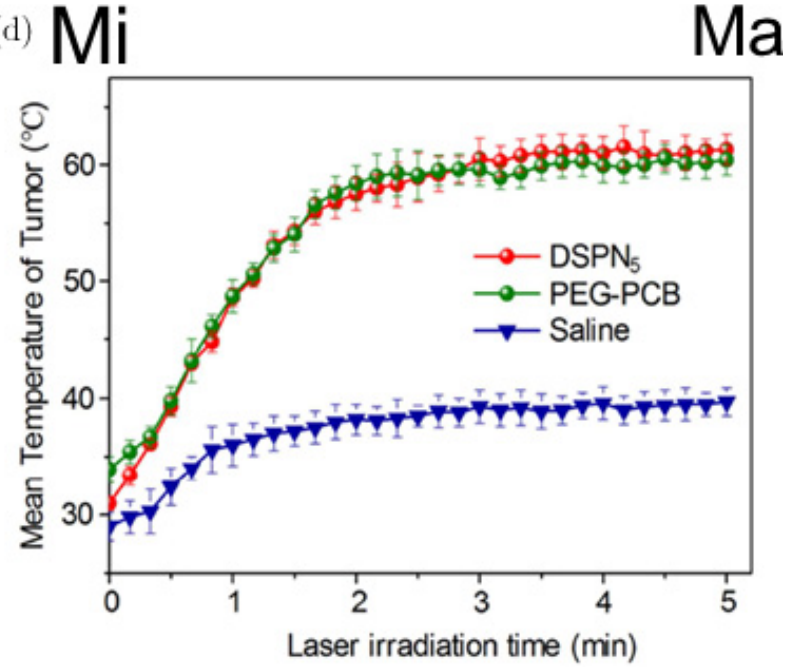

Source: Reference 43, Copyright 2017, Elsevier.

Fig. 6. PAI and FLI of tumor on living mice after the injection of PEG-PCB CPNs and tumor temperature evolution after injection PEG-PCB CPNs at laser irradiation.

PTT and chemotherapy demonstrates a higher therapeutic efficacy. Recently Pu's group utilized PEG-PCB CPNs for NIR FLI and PAI. ${ }^{43}$ And this diagnostic probe can also interact with doxorubicin (DOX) for drug encapsulation and delivery. Therefore, PEG-PCB CPNs can serve as a therapeutic platform with multimodal imaging and therapeutic capacity (Fig. 6) although there are still undesired side effects on normal tissues.

\subsection{Conjugated polymer nanoparticles for PTT and photodynamic therapy}

Interestingly, CPNs have the large absorption interface, which can be easily modified to design new photosensitizers for photodynamic therapy (PDT). The energy from conjugated polymer nanoparticles is able to be transferred efficiently to photosensitizer for increased yield of singlet oxygen. Therefore, PAI guided PTT/PDT is a very promising strategy, which can simultaneously improve the treatment efficacy and detection accuracy of cancer. Dong et al. synthesized a D-A-D structured DPP-TPA CPNs for PAI guided PTT and PDT (Fig. 7). ${ }^{44}$ Owing to the D-A-D structure of DPP-TPA CPNs, the charge generation and transportation can be greatly enhanced, leading to a high photothermal conversion efficiency $(\eta=34.5 \%)$ and excellent ROS generation $(\Phi \Delta=33.6 \%)$. In addition DPPTPA CPNs also exhibits strong PA signals, which can serve as the contrast agent for enhanced PAI. 
(a)
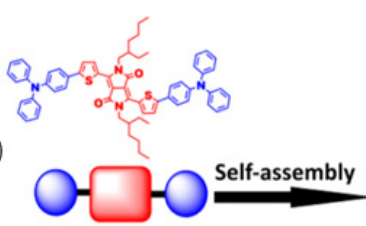

D-A-D structured DPP-TPA

$\mathrm{O}=$ Electron donor

$\square=$ Electron acceptor

(b)

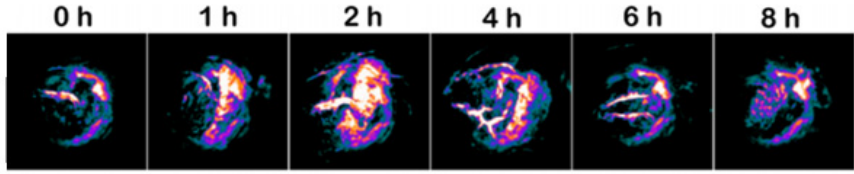

(c)
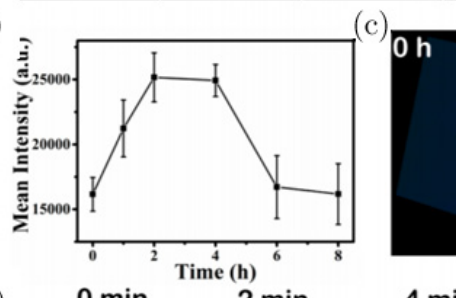

(d)

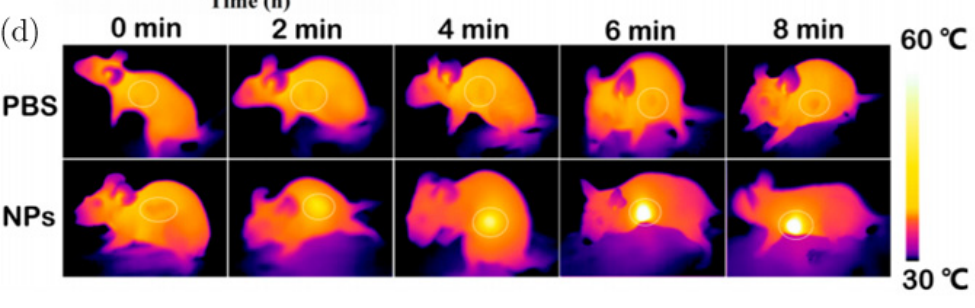

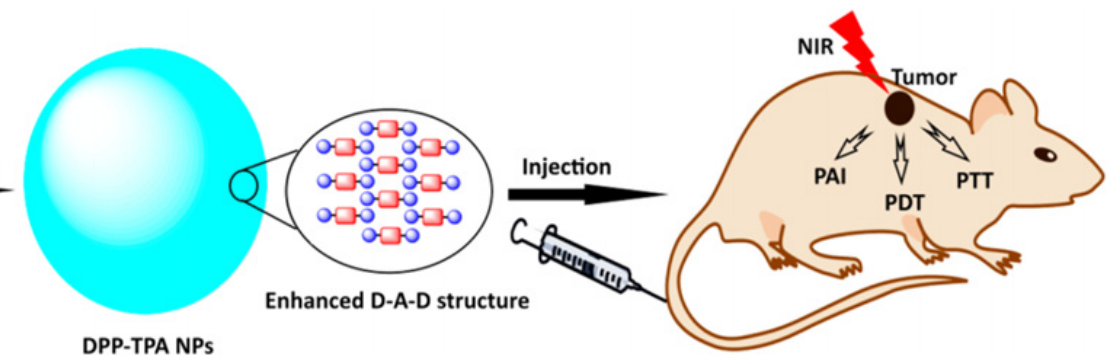

(e)

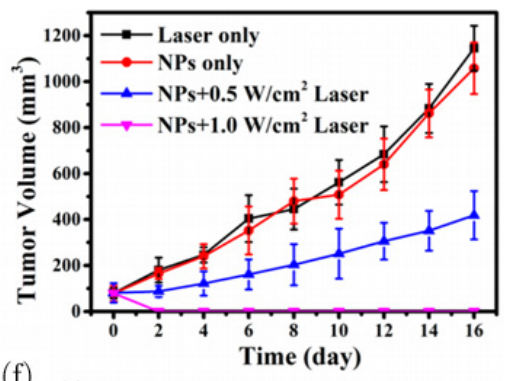

(f)

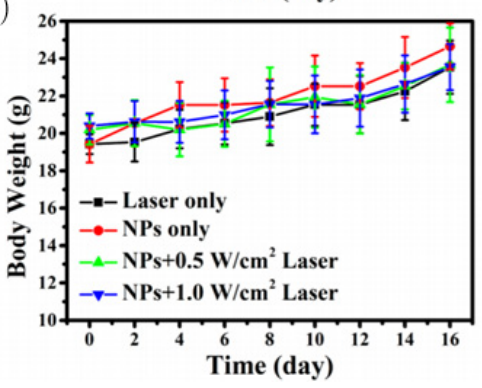

Source: Reference 44, Copyright 2017, American Chemical Society.

Fig. 7. (a)-(f) DPP-TPA CPNs for PAI-Guided PDT/PTT and the therapeutic effect of tumor sites after injecting DPP-TPA CPNs.

\section{Conclusion}

The development of a nano-diagnostic preparation that integrates imaging diagnosis and treatment will reduce the number of treatments for patients and improve the efficiency of tumor treatment. Multifunctional organic CPNs have shown the great promises for cancer theranostics due to their excellent physical and chemical properties as well as attractive biocompatibility. The excellent biocompatibility is a prerequisite for clinical application of produced nanoprobes. From this point of view, CPNs are purely organic materials and there is no potential concern on their long-term biotoxicity as compared to inorganic quantum dots and up-conversion nanoparticles. PAI is an emerging hybrid imaging modality based on photoacoustic effect, which has exhibited its advantages for highresolution molecular imaging with excellent penetration depth. Therapeutic techniques including PTT, PDT and chemotherapy under the guidance of PAI have provided us novel strategies for accurate diagnosis and precise treatment of tumors. It is expected that PA-based multimodal imaging and treatment based on these multifunctional CPNs can pave a new avenue for improved cancer theranostics in the future.

\section{Acknowledgments}

We acknowledge financial support from grants MYRG2014-00093-FHS, MYRG 2015-00036-FHS, MYRG2016-00110-FHS and MYRG2018-00081FHS from the University of Macau in Macau and grants FDCT 0011/2018/A1 and FDCT 025/2015/ A1 from the Macau government.

\section{References}

1. Y. Y. Wu, H. F. Ruan, R. Zhao, Z. Z. Dong, W. H. Li, X. J. Tang, J. H. Yuan, X. H. Fang, "Ultrastable 
fluorescent polymer dots for stimulated emission depletion bioimaging," Adv. Opt. Mater. 6, 1800333 (2018).

2. M. Nurunnabi, Z. Khatun, G. R. Reeck, D. Y. Lee, Y. K. Lee, "Near infra-red photoluminescent graphene nanoparticles greatly expand their use in noninvasive biomedical imaging," Chem. Commun. 49, 5079-5081 (2013).

3. M. E. Tanenbaum, L. A. Gilbert, L. S. Qi, J. S. Weissman, R. D. Vale, "A protein-tagging system for signal amplification in gene expression and fluorescence imaging," Cell 159, 635-646 (2014).

4. J. Panes, R. Bouzas, M. Chaparro, V. GarciaSanchez, J. P. Gisbert, B. M. de Guerenu, J. L. Mendoza, J. M. Paredes, S. Quiroga, T. Ripolles, J. Rimola, "Systematic review: The use of ultrasonography, computed tomography and magnetic resonance imaging for the diagnosis, assessment of activity and abdominal complications of Crohn's disease," Aliment. Pharmacol. Ther. 34, 125-145 (2011).

5. M. Infante, S. Cavuto, F. R. Lutman, E. Passera, M. Chiarenza, G. Chiesa, G. Brambilla, E. Angeli, G. Aranzulla, A. Chiti, M. Scorsetti, P. Navarria, R. Cavina, M. Ciccarelli, M. Roncalli, A. Destro, E. Bottoni, E. Voulaz, V. Errico, G. Ferraroli, G. Finocchiaro, L. Toschi, A. Santoro, M. Alloisio, D. S. Grp, "Long-term follow-up results of the dante trial, a randomized study of lung cancer screening with spiral computed tomography," Am. J. Respir. Crit. Care Med. 191, 1166-1175 (2015).

6. N. Tahara, J. Mukherjee, H. J. de Haas, A. D. Petrov, A. Tawakol, N. Haider, A. Tahara, C. C. Constantinescu, J. Zhou, H. H. Boersma, T. Imaizumi, M. Nakano, A. Finn, Z. Fayad, R. Virmani, V. Fuster, L. Bosca, J. Narula, "2-deoxy-2[F-18]fluoro-D-mannose positron emission tomography imaging in atherosclerosis," Nat. Med. 20, 215-219 (2014).

7. H. Yoneyama, K. Nakajima, K. Okuda, S. Matsuo, M. Onoguchi, S. Kinuya, L. Edenbrandt, "Reducing the small-heart effect in pediatric gated myocardial perfusion single-photon emission computed tomography," J. Nucl. Cardiol. 24, 1378-1388 (2017).

8. J. Q. Chen, C. B. Liu, D. H. Hu, F. Wang, H. W. Wu, X. J. Gong, X. Liu, L. Song, Z. H. Sheng, H. R. Zheng, "Single-Layer MoS2 Nanosheets with amplified photoacoustic effect for highly sensitive photoacoustic imaging of orthotopic brain tumors," Adv. Funct. Mater. 26, 8715-8725 (2016).

9. Y. Y. Jiang, K. Y. Pu, "Advanced photoacoustic imaging applications of near-infrared absorbing organic nanoparticles," Small 13, 1700710 (2017).
10. F. Gong, L. Cheng, N. L. Yang, Q. T. Jin, L. L. Tian, M. Y. Wang, Y. G. Li, Z. Liu, "Bimetallic oxide mnmoox nanorods for in vivo photoacoustic imaging of gsh and tumor-specific photothermal therapy," Nano Lett. 18, 6037-6044 (2018).

11. Y. D. Jin, C. X. Jia, S. W. Huang, M. O'Donnell, X. H. Gao, "Multifunctional nanoparticles as coupled contrast agents," Nat. Commun. 1, 41 (2010).

12. S. Mallidi, G. P. Luke, S. Emelianov, "Photoacoustic imaging in cancer detection, diagnosis, and treatment guidance," Trends. Biotechnol. 29, 213-221 (2011).

13. A. C. Chen, S. Chatterjee, "Nanomaterials based electrochemical sensors for biomedical applications," Chem. Soc. Rev. 42, 5425-5438 (2013).

14. H. L. Qi, Y. Peng, Q. Gao, C. X. Zhang, "Applications of nanomaterials in electrogenerated chemiluminescence biosensors," Sensors 9, 674-695 (2009).

15. P. C. Ray, "Size and shape dependent second order nonlinear optical properties of nanomaterials and their application in biological and chemical sensing," Chem. Rev. 110, 5332-5365 (2010).

16. B. Xu, Y. N. Zhao, Z. F. Xiao, B. Wang, H. Liang, X. Li, Y. X. Fang, S. F. Han, X. R. Li, C. X. Fan, J. W. Dai, "A dual functional scaffold tethered with egfr antibody promotes neural stem cell retention and neuronal differentiation for spinal cord injury repair," Adv. Healthc. Mater. 6, 1601279 (2017).

17. Y. Ni, R. K. Kannadorai, J. J. Peng, S. W. K. Yu, Y. T. Chang, J. S. Wu, "Naphthalene-fused BODIPY near-infrared dye as a stable contrast agent for in vivo photoacoustic imaging," Chem. Commun. 52, 11504-11507 (2016).

18. J. C. Ge, Q. Y. Jia, W. M. Liu, L. Guo, Q. Y. Liu, M. H. Lan, H. Y. Zhang, X. M. Meng, P. F. Wang, "Red-emissive carbon dots for fluorescent, photoacoustic, and thermal theranostics in living mice," Adv. Mater. 27, 4169-4177 (2015).

19. R. Cheheltani, R. M. Ezzibdeh, P. Chhour, K. Pulaparthi, J. Kim, M. Jurcova, J. C. Hsu, C. Blundell, H. I. Litt, V. A. Ferrari, H. R. Allcock, C. M. Sehgal, D. P. Cormode, "Tunable, biodegradable gold nanoparticles as contrast agents for computed tomography and photoacoustic imaging," Biomaterials 102, 87-97 (2016).

20. G. X. Lv, W. S. Guo, W. Zhang, T. B. Zhang, S. Y. Li, S. Z. Chen, A. S. Eltahan, D. L. Wang, Y. Q. Wang, J. C. Zhang, P. C. Wang, J. Chang, X. J. Liang, "Near-infrared emission cuins/zns quantum dots: All-in-one theranostic nanomedicines with intrinsic fluorescence/photoacoustic imaging for tumor phototherapy," ACS Nano 10, 9637-9645 (2016). 
21. S. K. Maji, S. Sreejith, J. Joseph, M. J. Lin, T. C. He, Y. Tong, H. D. Sun, S. W. K. Yu, Y. L. Zhao, "Upconversion nanoparticles as a contrast agent for photoacoustic imaging in live mice," Adv. Mater. 26, 5633-5638 (2014).

22. J. L. Grimland, C. F. Wu, R. R. Ramoutar, J. L. Brumaghim, J. McNeill, "Photosensitizer-doped conjugated polymer nanoparticles with high crosssections for one- and two-photon excitation," Nanoscale 3, 1451-1455 (2011).

23. H. B. Chen, H. Zhou, X. J. Men, K. Sun, Z. Z. Sun, X. F. Fang, C. F. Wu, "Light-induced pegylation and functionalization of semiconductor polymer dots," ChemNanoMat 3, 755-759 (2017).

24. X. Z. Chen, Z. H. Liu, R. Q. Li, C. Y. Shan, Z. P. Zeng, B. X. Xue, W. H. Yuan, C. Mo, P. Xi, C. F. $\mathrm{Wu}, \mathrm{Y}$. J. Sun, "Multicolor super-resolution fluorescence microscopy with blue and carmine small photoblinking polymer dots," ACS Nano 11, 80848091 (2017).

25. J. L. Geng, C. C. Goh, N. Tomczak, J. Liu, R. R. Liu, L. Ma, L. G. Ng, G. G. Gurzadyan, B. Liu, "Micelle/Silica Co-protected Conjugated polymer nanoparticles for two-photon excited brain vascular imaging," Chem. Mater. 26, 1874-1880 (2014).

26. C. F. Wu, D. T. Chiu, "Highly Fluorescent semiconducting polymer dots for biology and medicine," Angew. Chem. Int. Edit. 52, 3086-3109 (2013).

27. K. W. Chang, Y. B. Liu, D. H. Hu, Q. F. Qi, D. Y. Gao, Y. T. Wang, D. L. Li, X. J. Zhang, H. R. Zheng, Z. H. Sheng, Z. Yuan, "Highly stable conjugated polymer dots as multifunctional agents for photoacoustic imaging-guided photothermal therapy," ACS Appl. Mater. Inter. 10, 7012-7021 (2018).

28. P. Huang, J. Lin, W. W. Li, P. F. Rong, Z. Wang, S. J. Wang, X. P. Wang, X. L. Sun, M. Aronova, G. Niu, R. D. Leapman, Z. H. Nie, X. Y. Chen, "Biodegradable gold nanovesicles with an ultrastrong plasmonic coupling effect for photoacoustic imaging and photothermal therapy," Angew. Chem. Int. Edit. 52, 13958-13964 (2013).

29. K. Pu, J. G. Mei, J. V. Jokerst, G. S. Hong, A. L. Antaris, N. Chattopadhyay, A. J. Shuhendler, T. Kurosawa, Y. Zhou, S. S. Gambhir, Z. N. Bao, J. H. Rao, "Diketopyrrolopyrrole-based semiconducting polymer nanoparticles for in vivo photoacoustic imaging," Adv. Mater. 27, 5184-5190 (2015).

30. J. Liu, J. L. Geng, L. D. Liao, N. Thakor, X. H. Gao, B. Liu, "Conjugated polymer nanoparticles for photoacoustic vascular imaging," Polym. Chem. 5, 2854-2862 (2014).

31. K. Li, B. Liu, "Polymer-encapsulated organic nanoparticles for fluorescence and photoacoustic imaging," Chem. Soc. Rev. 43, 6570-6597 (2014).
32. J. C. Li, J. H. Rao, K. Y. Pu, "Recent progress on semiconducting polymer nanoparticles for molecular imaging and cancer phototherapy," Biomaterials 155, 217-235 (2018).

33. L. H. Feng, C. L. Zhu, H. X. Yuan, L. B. Liu, F. T. Lv, S. Wang, "Conjugated polymer nanoparticles: Preparation, properties, functionalization and biological applications," Chem. Soc. Rev. 42, 6620-6633 (2013).

34. H. B. Chen, J. Zhang, K. W. Chang, X. J. Men, X. F. Fang, L. B. Zhou, D. L. Li, D. Y. Gao, S. Y. Yin, X. J. Zhang, Z. Yuan, C. F. Wu, "Highly absorbing multispectral near-infrared polymer nanoparticles from one conjugated backbone for photoacoustic imaging and photothermal therapy," Biomaterials 144, 42-52 (2017).

35. J. Zhang, H. B. Chen, T. Zhou, L. M. Wang, D. Y. Gao, X. J. Zhang, Y. B. Liu, C. F. Wu, Z. Yuan, "A PIID-DTBT based semi-conducting polymer dots with broad and strong optical absorption in the visible-light region: Highly effective contrast agents for multiscale and multi-spectral photoacoustic imaging," Nano Res. 10, 64-76 (2017).

36. J. Liu, X. L. Cai, H. C. Pan, A. Bandla, C. K. Chuan, S. W. Wang, N. Thakor, L. D. Liao, B. Liu, "Molecular engineering of photoacoustic performance by chalcogenide variation in conjugated polymer nanoparticles for brain vascular imaging," Small 14, 1703732 (2018).

37. X. Zhen, X. H. Feng, C. Xie, Y. J. Zheng, K. Y. Pu, "Surface engineering of semiconducting polymer nanoparticles for amplified photoacoustic imaging," Biomaterials 127, 97-106 (2017).

38. C. Xie, P. K. Upputuri, X. Zhen, M. Pramanik, K. Y. Pu, "Self-quenched semiconducting polymer nanoparticles for amplified in vivo photoacoustic imaging," Biomaterials 119, 1-8 (2017).

39. D. Y. Gao, P. F. Zhang, Y. B. Liu, Z. H. Sheng, H. J. Chen, Z. Yuan, "Protein-modified conjugated polymer nanoparticles with strong near-infrared absorption: A novel nanoplatform to design multifunctional nanoprobes for dual-modal photoacoustic and fluorescence imaging," Nanoscale 10, 1974219748 (2018).

40. C. Xie, X. Zhen, Q. L. Lei, R. Ni, K. Y. Pu, "Selfassembly of semiconducting polymer amphiphiles for in vivo photoacoustic imaging," Adv. Funct. Mater. 27, 1605397 (2017).

41. Z. Yang, W. P. Fan, W. Tang, Z. Y. Shen, Y. L. Dai, J. B. Song, Z. T. Wang, Y. Liu, L. S. Lin, L. L. Shan, Y. J. Liu, O. Jacobson, P. F. Rong, W. Wang, X. Y. Chen, "Near-infrared semiconducting polymer brush and ph/gsh-responsive polyoxometalate cluster hybrid platform for enhanced tumor-specific phototheranostcs," Angew. Chem. Int. Edit. 57, 14101-14105 (2018). 
42. B. Guo, Z. H. Sheng, D. H. Hu, C. B. Liu, H. R. Zheng, B. Liu, "Through scalp and skull nir-ii photothermal therapy of deep orthotopic brain tumors with precise photoacoustic imaging guidance," $A d v$. Mater. 30, 1802591 (2018).

43. Y. Y. Jiang, D. Cui, Y. Fang, X. Zhen, P. K. Upputuri, M. Pramanik, D. Ding, K. Y. Pu, "Amphiphilic semiconducting polymer as multifunctional nanocarrier for fluorescence/photoacoustic imaging guided chemo-photothermal therapy," Biomaterials 145, 168-177 (2017).

44. Y. Cai, P. P. Liang, Q. Y. Tang, X. Y. Yang, W. L. Si, W. Huang, Q. Zhang, X. C. Dong, "Diketopyrrolopyrrole-triphenylamine organic nanoparticles as multifunctional reagents for photoacoustic imaging-guided photodynamic/photothermal synergistic tumor therapy," ACS Nano 11, 1054-1063 (2017). 